\title{
Advanced Esophageal Carcinoma
}

National Cancer Institute

\section{Source}

National Cancer Institute. Advanced Esophageal Carcinoma. NCI Thesaurus. Code C160599.

Esophageal carcinoma that has spread extensively to other anatomic sites or is no longer responding to treatment. 